\title{
Some connective tissue disorders of the lung
}

\author{
Margaret Turner-Warwick
}

Cardiothoracic Institute, University of London, Fulham Road, London SW3 6HP, UK.

\begin{abstract}
Summary: Many connective tissue disorders involve the lungs. The same clinical syndrome may be associated with several distinctive types of pathology in different patients. Fibrosing alveolitis is a common feature of a number of different syndromes. An hypothesis is set out in schematic form which may help to account for some of these differences and emphasizes the potential importance of the pulmonary vasculature in pathogenesis.
\end{abstract}

\section{Introduction}

The tissues of the lung alone receive virtually the entire venous circulating blood volume coming from other organs of the body. It is not therefore surprising that the lungs are affected in a wide variety of multisystem disorders. In particular it is not suprising that an inflammatory response and fibrotic reaction in the lung is common to several different connective tissue syndromes including systemic lupus erythematosus (SLE), rheumatoid arthritis and systemic sclerosis as well as occurring occasionally in a number of other conditions such as primary biliary cirrhosis, chronic active hepatitis, ulcerative colitis, Sjögren's syndrome, autoimmune thyroiditis and ill defined digital vasculitides. A central unresolved question is why lung involvement is very common in certain syndromes such as systemic sclerosis and apparently very much less common in others such as ulcerative colitis and perhaps chronic hepatic disease. Another unexplained fact is that a number of different types of pathology may be found in the lungs of different patients with the same connective tissue syndrome (Table I). For example, in rheumatoid arthritis,

Table I The overlap of pulmonary pathology in various different connective tissue disorders

\begin{tabular}{lccc}
\hline & SLE & $\begin{array}{c}\text { Systemic } \\
\text { sclerosis }\end{array}$ & $\begin{array}{c}\text { Rheumatoid } \\
\text { arthritis }\end{array}$ \\
\hline Pulmonary haemorrhage & + & - & - \\
Acute alveolitis & + & - & \pm \\
$\begin{array}{l}\text { Pleural effusions } \\
\text { Fibrosing alveolitis }\end{array}$ & ++ & - & + \\
Necrobiotic nodules & ++ & +++ & ++ \\
$\begin{array}{l}\text { Obliterative } \\
\text { bronchiolitis }\end{array}$ & - & - & + \\
\hline
\end{tabular}

Correspondence: Professor M. Turner-Warwick, Ph.D., D.Sc.(Hon), D.M., F.R.C.P., F.R.A.C.P., F.A.C.P.(Hon). necrotic nodules, pleural effusions, alveolitis or rarely pulmonary hypertension may each be the predominating lesion in different patients.

Another current unresolved argument centres on whether 'cryptogenic' fibrosing alveolitis (CFA) occurring in association with connective tissue disorders differs from 'lone' CFA (i.e. that condition apparently affecting the lungs only) either in its natural history or in its response to treatment.

The question has been considered in a large $\varnothing$ retrospective series of 139 patients with 'lone' CFA, comparing them to 66 patients with associated connective tissue diseases and followed during treatment over a similar period of time. ${ }^{1}$ The clinical features at presentation were found to be very similar although there was a trend towards a greater male predominance in those with 'lone' CFA. In particular, the severity of symptoms was notably similar. From this series about two thirds of each group was selected on clinical grounds for treatment with corticosteroids and a similar proportion showed a subjective improvement when assessed after 4 to 8 weeks, although there was a trend towards a better response in those with associated disease. Overall, however, the survival curves were almost superimposable even in those patients who had been followed for up to 15 years (Figure 1).

In spite of the many clinical similarities shown between CFA with and without associated disorders, some of the immunological characteristics differ. Circulating non-organ specific autoantibodies are increased in both groups but the frequency is higher in those with associated disease. Some differences are also found in the type of nuclear antibodies demonstrated. For example Chapman and colleagues $^{2}$ showed that extractable anti-ribonuclear protein antibody was found in only $4 \%$ of 689 patients with 'lone' CFA but in $12(22 \%)$ of 54 patients with associated connective tissue disorders.

(C) The Fellowship of Postgraduate Medicine, 1988 


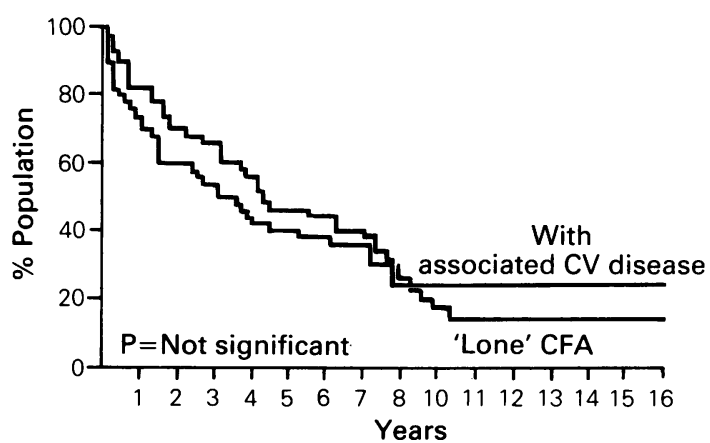

Figure 1 Log rank survival curves for cryptogenic fibrosing alveolitis (CFA) with and without associated collagen vascular $(\mathrm{CV})$ disease.
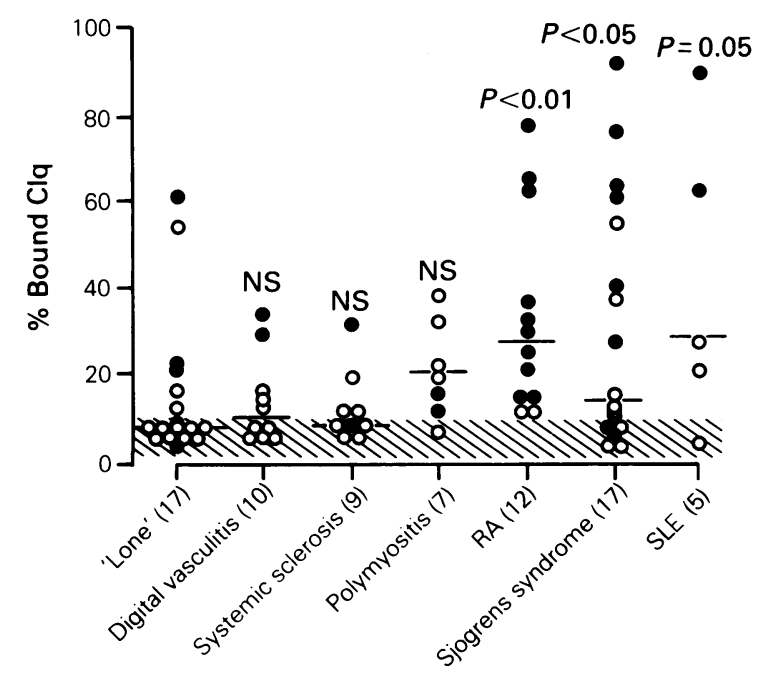

Figure $2 \mathrm{C} 1 \mathrm{q}$ binding studies in 77 patients with cryptogenic fibrosing alveolitis. Positive rheumatoid factor; $\bigcirc$, negative rheumatoid factor.

In passing, it should be noted that in this series extractable nuclear antigen (ENA) was found in association with a variety of clinical syndromes and the clinical features of 'mixed connective tissue disease', as described by Sharpe and colleagues ${ }^{3}$ was found in only two patients.

As expected rheumatoid factor is raised more frequently and found in higher titres in those with associated disorders because this group includes all patients with rheumatoid arthritis. However, raised titres of rheumatoid factor are found more frequently in patients with 'lone' CFA compared with normal controls. ${ }^{10}$

Circulating immune complexes are commonly found in CFA but the prevalence as well as the titres are often higher in those with associated disorders (Figure 2). Probably associated with this, antibody-dependent lymphocyte cytoxicity shows greater impairment in CFA with associated disorders than in those with 'lone' disease. ${ }^{4}$ When fibrosing alveolitis is also present, deposits of $c$ immune complexes in the pulmonary capillaries have been shown occasionally both in SLE where complement can also be found (Figure 3) and in rheumatoid arthritis where it is sometimes absent. ${ }^{5,6}$ Deposits in the media of larger pulmonary arteries are very occasionally seen (Figure 4$).{ }^{7}$ Electron microscopic evidence of immune complexes is almost always negative although occasionally tubular reticular structures as previously described by Yeo and colleagues ${ }^{8}$ are found (Figure 5).

The fact that when CFA with and without associated disorders are compared, several immunological features differ (at least in intensity) while the clinical features are similar, suggests that these immunological markers may have relatively little influence on the pathogenesis of disease in the lung.

\section{Pathogenesis}

To explain the similar as well as distinctive patholoe gies found in the lungs in different connective tissue disorders, we have explored the hypothesis repres. sented schematically in Figure 6 . In this it suggested that the type of lesion found is at least in part due to the size of the pulmonary vessels bearing the brunt of damage as well as the modulating influence of the secondary circulation of the lung (bronchial circulation), 'protecting' tissue oxygenation. The larger the vessels affected the greater the chances of ischaemic necrosis or infarction. Microscopic areas of increased metabolism due to concomitant inflammatory infiltrates may be particularly vulnerable.

The hypothesis goes some way to explain why a similar pathological process in the lung may be found in a range of disorders limited to the lung, such as 'lone' CFA, pulmonary hypertension, idiopathic pulmonary haemosiderosis (IPH) and haemorrhage or veno-occlusive disease. It may also explain why an identical pathology is also found in the lungs of patients with a variety of associated connective tissue disorders. For example CFA, pulmonary hypertension and a pulmonary haemosiderosis and haemorrhage have also all been found in patients with SLE. Evidence supporting the importance of pulmonary capillary damage in fibrosing alveolitis with and without associated connective tissue disease is to be found in the marked increase in lung permeability measurements observed in these conditions (Figure 7). Permeability is even 


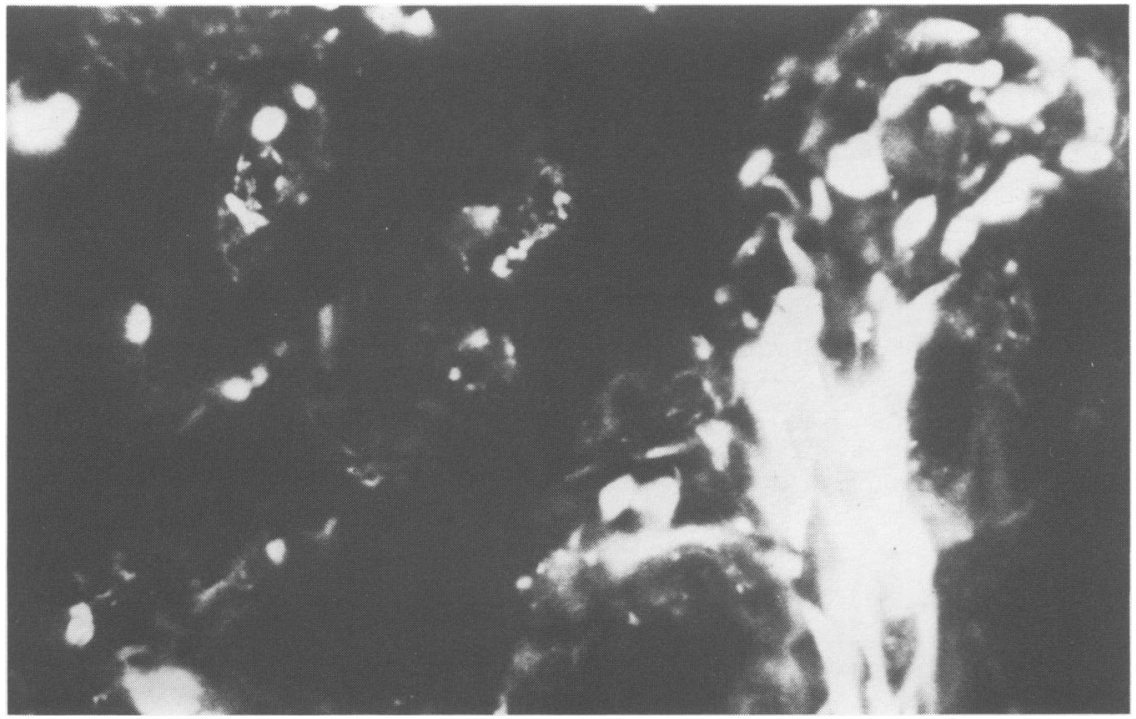

Figure 3 Lung biopsy for a patient with SLE and fibrosing alveolitis. Fluorescein labelled antibody to complement C3 showing granular deposits in the alveolar walls. Dense white areas on the right hand side of the photograph are autofluorescent elastin.

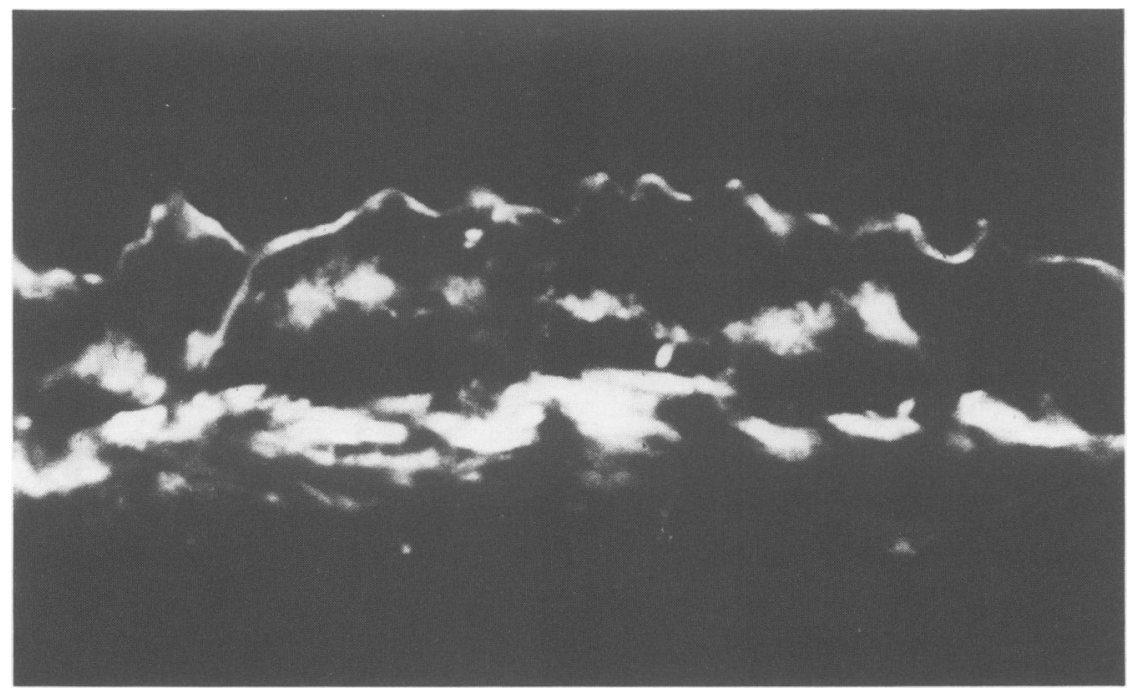

Figure 4 A pulmonary artery from the same preparation as Figure 3 showing complement deposits between the internal and external elastic laminae.

more rapid in cases of IPH and pulmonary haemorrhage where evidence for pulmonary endothelial damage on electron microscopy has been reported. ${ }^{9}$

Alveolitis is also found in 'hypersensitivity' drug reactions and these too may affect other systems commonly involved in connective tissue disorders including joints, serosal surfaces, skin including digital vasculitis, eyes and kidneys. The question as to whether the pulmonary capillaries are a primary target or are involved as a secondary manifestation of an inflammatory response is not of course resolved, but in view of the clinical patterns of disease, the hypothesis that the primary damage is vascular is at least tenable. 

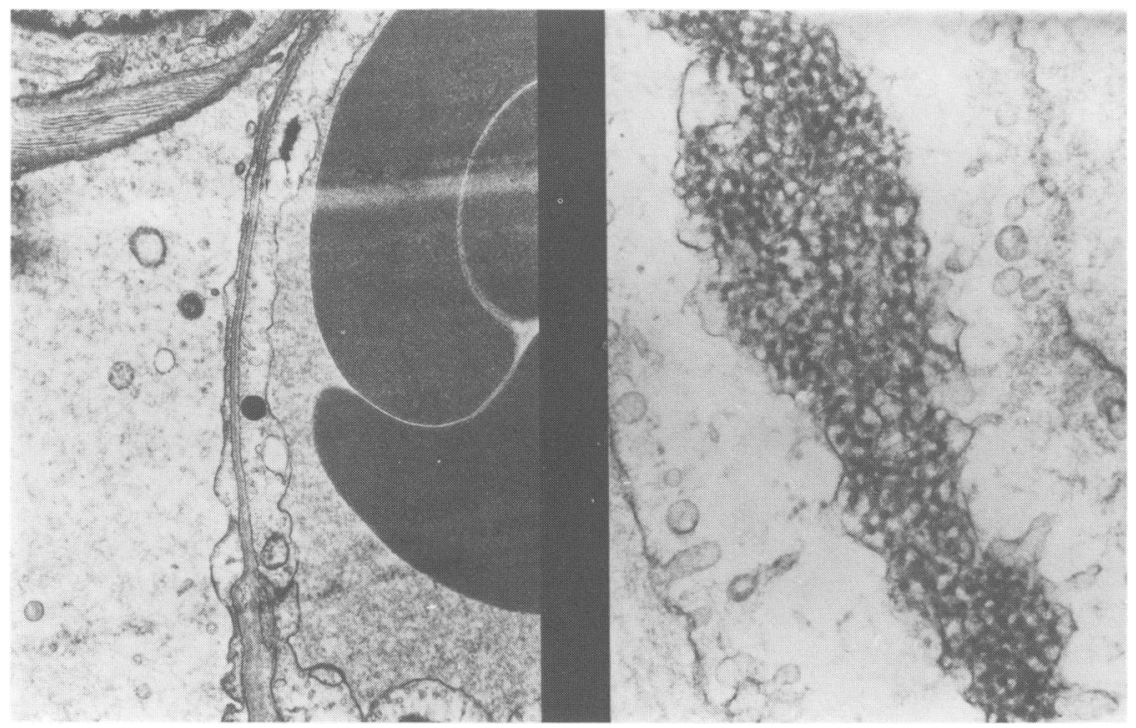

Figure 5 Electron dense deposits in the pulmonary capillary endothelial cells seen in low and high magnification.

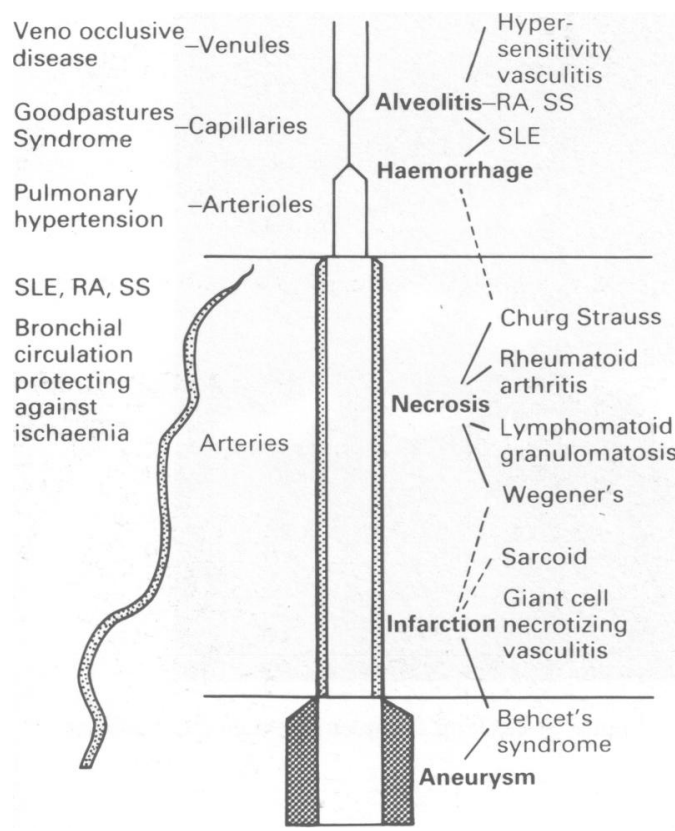

Figure 6 A diagram to illustrate the hypothesis that damage to different sizes of blood vessels in the lung in connective tissue disorders accounts for some of the distinctive pathologies observed as well as those found in common. It also goes some way to explain the overlap between 'lone' pulmonary disorders and similar pathologies seen in a variety of multisystem disorders (see text for further explanation).

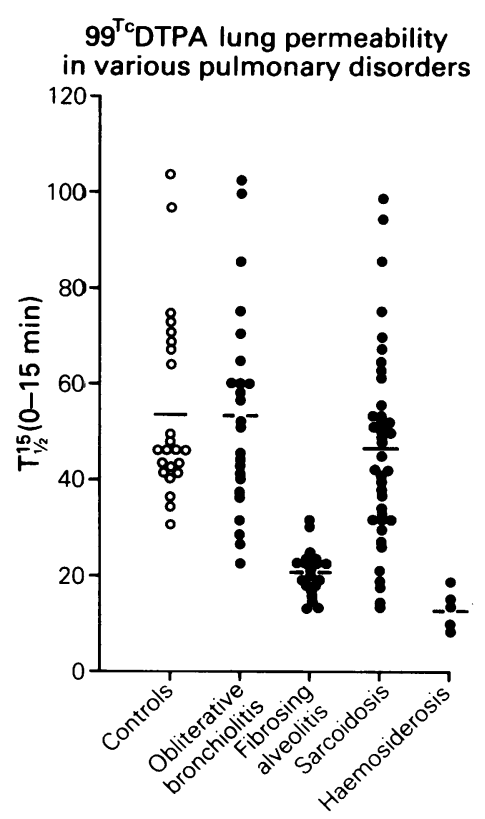

Figure 7 Lung permeability measurements in CFA, sarcoidosis, obliterative bronchiolitis and idiopathic pulmonary haemosiderosis. 
Where larger vessels are affected or where larger areas of inflammation are not associated with a corresponding angiogenesis and cannot be reached for anatomical reasons by the bronchial circulation, ischaemic necrosis may develop. Such a hypothesis could contribute to explain the necrotic lesions seen in rheumatoid arthritis, Wegener's granuloma, and the rare syndrome of necrotizing sarcoidosis - in all of which there is good evidence of an associated vasculitis of vessels larger than capillaries. Where even larger vessels are involved infarction may be found as in Behçet's disease ${ }^{10}$ and some patients with SLE.

\section{Evidence of host susceptibility to lung involvement in connective tissue disorders}

An explanation must be sought for the fact that only a small proportion of patients with certain connective tissue disorders develop any type of lung involvement. Many years ago, Tomasi and colleagues $^{11}$ suggested that those patients with especially high titres of rheumatoid factor were more likely to develop fibrosing alveolitis. An early study of our patients with rheumatoid arthritis with and without fibrosing alveolitis showed that the distribution of rheumatoid factor was in fact very similar. ${ }^{12}$ Indirect evidence suggestive of some susceptibility is, however, indicated by the finding that over $70 \%$ of a series of 39 patients with fibrosing alveolitis and rheumatoid arthritis developed lung involvement within 4 years of the onset of joint symptoms. Conversely, of those rare cases where the lungs were involved first, the joints were affected within 3 years. ${ }^{13}$ Thus CFA seems to appear as a relatively early manifestation of rheumatoid disease in a predisposed sub-group of patients. The markers for this susceptibility towards lung involvement have not yet been identified.

Perhaps the most clear-cut evidence of an identifiable susceptibility is provided by the marker, histidyl t-RNA synthetase (anti-Jo-1 antibody) in polymyositis. Bernstein and colleagues ${ }^{14}$ have recently shown that this antibody was present in less than $5 \%$ of patients with polymyositis or CFA alone, but was observed in $77 \%$ of patients with the combined syndrome. This finding suggests that analogous markers might be sought in other conditions. Such markers might then be used to establish whether a genetic predisposition is important in the development of lung disease. This in turn might also give us more insight into the nature of CFA when it occurs in the 'lone' form.

\section{The severity and type of fibrosing alveolitis observed}

It is often stated that alveolitis when it occurs in connective tissue disorders is mild and relatively non-progressive. In this respect, the chest radiograph may be misleadingly normal but computerized tomographic scanning (CT) of the lungs can be helpful in indicating the real severity of the disease (Figures 8 and 9). In SLE the severity of the lung involvement may be difficult to assess when there is substantial diaphragmatic or pleural involvement. Under these circumstances lung lavage may be useful to establish the co-existence of intrapulmonary lung involvement by demonstrating the presence of abnormal numbers of inflammatory cells.

A recent study has been completed on 30 consecutive patients with systemic sclerosis, 16 of whom

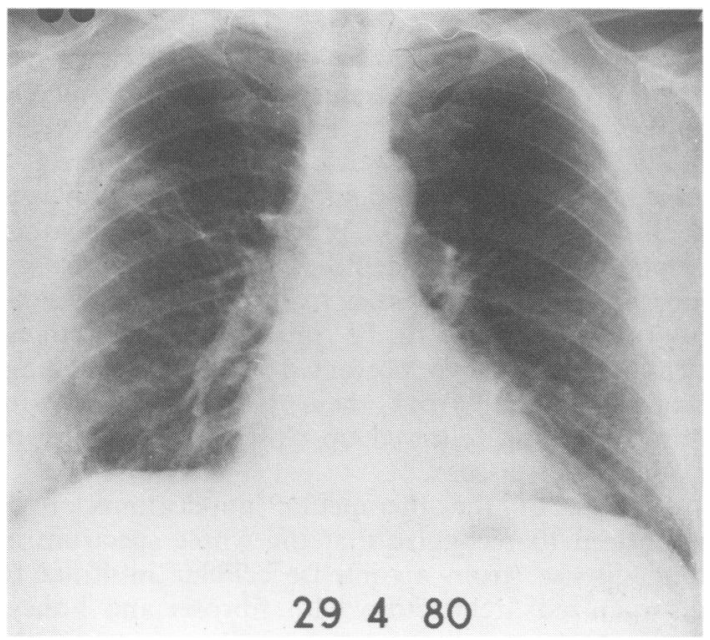

Figure 8 A virtually normal chest radiograph of a patient with SLE.

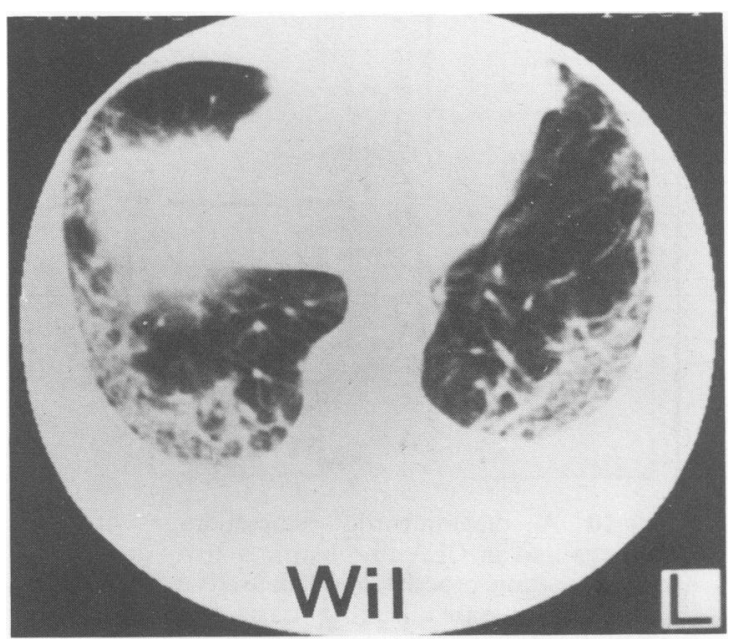

Figure 9 Computerized assisted tomography from the same patient showing the extent of the circumferential changes of fibrosing alveolitis. 
were asymptomatic and had normal chest radiographs. In an attempt to identify early disease, lavage, lung permeability measurements and CT scans were performed. Only 2 of these patients gave completely normal results. Lung lavage was abnormal in $11(73 \%)$ some showing excess lymphocytes and others neutrophils. Lung permeability was increased in $8(53 \%)$ and CT scans abnormal in 7 $(44 \%) .{ }^{15}$ In two cases where all 3 measurements were abnormal in the presence of a normal chest radiograph and lung function, lung biopsy showed a mixed pathology of fibrosis together with an inflammatory infiltrate. Thus, even in preclinical disease using conventional criteria, fibroblastic proliferation is already a feature. Thus the concept of an inflammatory alveolitis which only later develops into fibrotic response needs to be reconsidered. Rather, we suggest that even in disease of very limited extent (as observed on CT scans), there is a concomitant cellular inflammatory and fibroblastic proliferation (Figure 10). Whether an inflammatory response only can be identified in even earlier cases, identified by abnormalities of lavage and permeability only, remains to be proved. So far ethical considerations have prevented us from obtaining biopsy material from these patients. They are, however, being followed up closely for evidence of extension of disease.

Because of the therapeutic implications, it is important to recognize that the whole spectrum of lung disease (from an intense cellular infiltrate to disorganized architecture with fibrosis and honey-

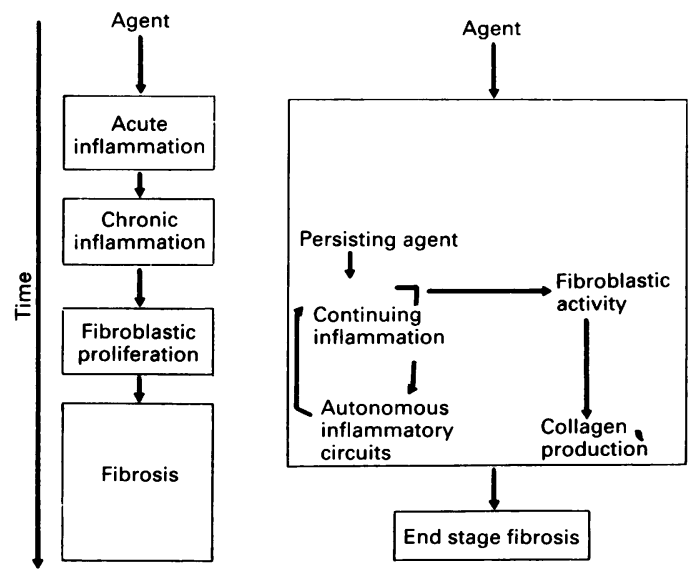

Figure 10 A diagrammatic representation of the hypothesis that in CFA fibroblastic activity and chronic inflammation proceed simultaneously but progress in extent of disease. This contrasts with the more traditional scheme outlined on the left which sets out progression in a serial way proceeding from inflammation to fibrosis. combing) may occur in association with any indivi气 dual connective tissue disease in which fibrosing alveolitis occurs. In particular, more acute 'cellularฉ̨ disease has been seen in systemic sclerosis and if treated at this stage considerable improvement has: been seen. Incidentally some of the highest lavage lymphocyte counts have been seen in systemice sclerosis. True vasculitis of the pulmonary vessels is uncommon but intimal thickening often associated with clinical evidence of pulmonary hypertension is found sometimes. Pulmonary hypertension withoutes associated alveolitis has also been described occa- $\overrightarrow{0}$ sionally in SLE, rheumatoid arthritis and systemic sclerosis. ${ }^{16-18}$ We have seen one most unusual case $\vec{\omega}$ with CREST syndrome and primary biliary cirrho $\frac{\Omega}{8}$ sis associated with severe organising pneumoniaco the latter responded dramatically to corticosteroids 3 . but severe pulmonary hypertension developed 3\$ years later in the absence of any relapse of the parenchymal lung disease.

\section{Some unusual syndromes}

\section{Obliterative bronchiolitis and rheumatoid arthritis}

This syndrome was described by Geddes and collea-co gues. ${ }^{19}$ Unlike CFA it often affects patients wiono longstanding rheumatoid arthritis but it is semn nevertheless in relatively young patients in theirs third and fourth decade. It can be distinguished금 from asthma by the lack of reversibility of airflown limitation by any therapy, from chronic bronchitis by the absence of cough and sputum and from destructive emphysema by the absence of radio-음 graphic supporting evidence and the presence of $a_{5}^{3}$ normal transfer factor for carbon monoxide when corrected for accessible lung volume (KCO).

Unlike other chronic airflow disease, obliterative bronchiolitis often starts abruptly and often runs $a_{-}$. fairly rapid downhill course and many deaths occur 3 within 2 or 3 years. On auscultation, crepitations are absent but a high pitched end inspiratory 'squeak'윽 is heard in some. This sign presumably reflects $\mathrm{O}$ intrinsic narrowing of smaller airways.

Histological examination confirms obliteration of $\frac{D}{O}$ the small airways by an intense inflammatory infiltrate, so that the lumen may be reduced to a slit or even disappear entirely. The almost completen absence of a small airway can easily be identified by the appearance of a normal small pulmonary artery which is not accompanied by an equal sized bronchiole. It should be emphasized that seriale sections are often necessary to display the full pathology. The aetiology of obliterative bronchioli-tis is unknown. A causative association with peni- $\frac{0}{3}$ cillamine therapy has been suggested but at least 5 유 
of the 20 cases recently reviewed never received this drug (Sweatman, unpublished). Whether penicillamine has some facilitating role in other cases is unclear, but this possibility is currently being examined in a case control study.

\section{Digital vasculitis and lung disease}

Occasionally patients are seen who develop severe digital vasculitis with necrosis of the finger tips in association with fibrosing alveolitis in the lung. ${ }^{20}$ Digital vasculitis of this type is well described in systemic sclerosis and rheumatoid arthritis. These associated conditions were present in 5 of 12 recently described cases. Two other cases were found in association with polymyositis and fibrosing alveolitis. However, of particular interest were 3 cases developing florid digital vasculitis in which the only other clinical manifestation was CFA. Circulating immune complexes were infrequent but IgM was often raised in both patients with and without arthritis. Corticosteroids did not improve the vasculitis in any of the 10 cases in which it was used and, in fact, vasculitis occurred during steroid treatment of the lung condition in 6 . On the other hand, treatment with immunosuppressant drugs (cyclophosphamide or azathioprine) improved the digital vasculitis in 4 of 6 patients and penicillamine improved the fingers in 4 of 5 patients so treated. While corticosteroids uniformly failed to control the digital features they frequently improved the lung disease. Thus distinct pathologies occurring simultaneously in the fingers and lung responded differentially to treatments; a fact that must be accounted for when attempting to form hypotheses about pathogenesis.

\section{Lung disease associated with anti-mitochondrial antibody}

At least six distinct lung pathologies have been seen in association with a high titre anti-mitochondrial antibody. These include acute transient 'pneumonitis', fibrosing alveolitis, relapsing organising pneumonia, sarcoid-like granulomas, pleural effusions and radiographic evidence of small pulmonary venous arterial shunts (pulmonary 'spiders').

Sarcoid-like granulomata of the lung associated with mitochondrial antibody are of special interest because they present a number of features which are atypical for either sarcoidosis or primary biliary cirrhosis. ${ }^{21}$ Our cases presented with pulmonary symptoms and a chest radiograph showing widespread nodular shadows which, on biopsy, revealed typical sarcoid-like granulomata. Enlargement of the liver was an incidental finding but the pathology of this was variable. In some, there were granulomata without evidence of inflammatory changes in the portal tracts and normal biliary ducts; in others there was some evidence of biliary tract involvement. Irrespective of the liver histology, the alkaline phosphatase was considerably raised in all, as were cytoplasmic liver enzymes. On the other hand the serum angiotensin converting enzyme was normal in one and raised in two cases tested before treatment. Treatment with corticosteroids cleared the lung lesions dramatically but serial studies on the liver have shown a trend towards increasing features of primary biliary cirrhosis with established cirrhosis in two. These cases would seem to occupy an analogous position to the duck billed platypus in the evolution of species in that, while extremely rare, they appear to occupy a crucial linking position between a multiorgan sarcoid-like response and immunological and some pathological features of primary biliary cirrhosis. When the pathogenesis of these two conditions eventually becomes clear, the explanation for these rare 'overlap' cases should become apparent.

Another, perhaps uniquely rare, case has been seen which showed features of CREST syndrome, histologically proven primary biliary cirrhosis and lung pathology of relapsing organising pneumonia. The lung lesions cleared dramatically on corticosteroids, but relapsed, involving a new site in the lung after 3 years, when the dose of prednisone had been reduced to $5 \mathrm{mg}$ on alternate days. This lesion too, improved when the dose of steroids was increased and later still, severe pulmonary hypertension developed in the absence of relapse of parenchymal lung disease.

That granulomatous lesions and organising pneumonia occurring in association with high titre antimitochondrial antibody in the same patient are not chance findings is supported by a recent case. The patient presented with severe breathlessness and widespread confluent shadows on the chest radiograph. These partially cleared spontaneously, but some nodular lesions persisted. Lung biopsy showed typical sarcoid-like granulomas in some areas but an organising pneumonia with intraalveolar fibroblastic proliferation, in others. High titre anti-mitochondrial antibody was present and histology of the liver showed some infiltration of inflammatory cells in the portal tracts. When the pathogenesis of primary biliary cirrhosis and sarcoidosis is eventually established it seems likely that ideas relevant to understanding the pathogenesis of cryptogenic organising pneumonia may also become apparent.

\section{Conclusions}

One of the most challenging features of connective 
tissue disorders affecting the lung is the dichotomous situation where on the one hand many distinct pulmonary disorders are found in different patients with the same systemic syndrome, but on the other hand, the same pulmonary pathology (notably fibrosing alveolitis) is found in patients with clinically distinctive connective tissue syndromes.

The precise pathological features of fibrosing alveolitis found in an individual patient will also depend upon the stage of disease. This ranges from a predominant chronic inflammation response to gross fibrotic lung destruction. Recognition of different stages of disease is of great therapeutic importance because early active disease (even when asymptomatic) is more likely to respond to treatment than late disease, where destruction of lung architecture will deny the opportunity for improvement by any form of medication. Thus the aspects of pathology described here relating to staging are

\section{References}

1. Turner-Warwick, M. Interstitial pulmonary fibrosis with and without associated collagen vascular disease. Am Rev Respir Dis 1981, 123: 73.

2. Chapman, J.R., Charles, P.P.J., Venables, P.J.W. et al. Definition and clinical relevance of antibodies to nuclear ribonuclear protein and other nuclear antigens in patients with cryptogenic fibrosing alveolitis. $\mathrm{Am}$ Rev Resp Dis 1984, 130: 439-443.

3. Sharp, G.C., Irvin, W.S., Tan, E.M., Gould, R.G. \& Hollman, H.R. Mixed connective tissue disease - an apparent distinct rheumatic disease syndrome associated with a specific antibody to an extractable nuclear antigen (ENA). Am J Med 1972, 52: 148-159.

4. Haslam, P.L., Allan, F., Watling, A.F., Barrett, C., Morris, L. \& Turner-Warwick, M. Impaired antibodydependent cell-mediated cytotoxicity in cryptogenic fibrosing alveolitis. Clin Exp Immunol 1982, 49: 59-66.

5. de Horatius, R.J.J., Abruzzo, J.L. \& Williams, R.C. Immunofluorescent and immunological studies in rheumatoid lung. Arch Int Med 1972. 129: 441-446.

6. Turner-Stokes, L. \& Turner-Warwick, M. Intrathoracic manifestations of SLE. Clin Rheum Dis 1982, 8: 229-242.

7. Eisenberg, H., Simmons, D.H. \& Barnett, E.V. Diffuse pulmonary interstitial disease - an immunohistory study. Chest 1979, 75 (suppl 2): 262-264.

8. Yeo, P.P.B. \& Sinnian, R. Lupus cor pulmonale with electron miscroscope and immunofluorescent antibody studies. Ann Rheum Dis 1975, 34: 457-460.

9. Turner-Warwick, M. \& Dewar, A. Pulmonary haemorrhage and idiopathic haemosiderosis. Clin Radiol 1982, 33: 361-370.

10. Efthimiou, J., Johnson, C., Spiro, S.G. \& TurnerWarwick, M. Pulmonary disease in Behçets syndrome. $Q J$ Med 1986, 58: 259-280.

11. Tomasi, T.B., Fudenberg, H.H. \& Finby, N. Possible relationship of rheumatoid factors and pulmonary disease. Am J Med 1962, 33, 243-248. of more practical importance to clinicians than the largely academic, semantic exercise of attaching diagnostic labels to individual patients having these rare, complex, fascinating and often unique combinations of features which defy any simple grouping as described in standard textbooks.

\section{Acknowledgements}

Some of the ideas propounded in this review were included in the Konrad Hiller Lecture delivered to the Royal Australasian College of Physicians.

I would like to acknowledge my many colleagues throughout the UK who have referred patients with fascinating and rare syndromes which have allowed me to develop some of the hypotheses proposed in this review. As ever I am also grateful to my junior staff for their patience, Dr Pat Haslam for running the laboratory for so many years and Professor Corrin for his collaboration on the weekly clinical/pathology ward rounds continued for more than a decade.

12. Turner-Warwick, M. \& Doniach, D. Autoantibody studies in interstitial pulmonary fibrosis. $\mathrm{Br}$ Med $\mathrm{J}$ 1965, 1: 886-891.

13. Turner-Warwick, $M . \&$ Courtenay-Evans, $R_{\cap}$ Pulmonary manifestations of rheumatoid disease. Clip Rheum Dis 1977, 3: 549-564.

14. Bernstein, R.M., Morgan, S.H., Chapman, J. et ak Anti Jo-1 antibody: a marker for myositis wit? interstitial lung disease. $B r$ Med J 1984, 289: 151-152.

15. Glanville, A.R., Harrison, N.K., Miller, A. et al. The assessment of pulmonary involvement in systemic sclerosis (SS). (in press).

16. Nair, S.S., Askeri, A.D., Popelka, C.G. \& Kleinerman, J.F. Pulmonary hypertension in systemic lupus erythematosus. Arch Int Med 1980, 140: 109-111.

17. Gardner, D.L., Duthie, J.J.R., McLeod, J. \& Allan, W.S.A. Pulmonary hypertension in rheumatoid arthritis. Report of a case with intimal sclerosis of the pulmonary and digital arteries. Scott Med J 1957, 2, 183-188.

18. Young, R.H. \& Mark, G.J. Pulmonary vascular changes in scleroderma. Am J Med 1978, 64, 998-1004.

19. Geddes, D.M., Corrin, B., Brewerton, D.A., Davies, R.J. \& Turner-Warwick, M. Progressive airways and obliteration in adults and its association with rheumatoid diseases. $Q J$ Med 1977, 184: 427-444.

20. Hodson, M.E., Haslam, P.L., Spiro, S.G. \& TurnerWarwick, M. Digital vasculitis in patients with cryptogenic fibrosing alveolitis. $\mathrm{Br} J$ Dis Chest 1984 78: $140-148$.

21. Fagan, E.A., Moore-Gillon, J.C. \& Turner-Warwick, M. Multiorgan granulomas and mitochondrial antibodies. $N$ Engl J Med 1983, 308: 572-575.

22. Davison, A.G. \& Epstein, O. Relapsing organizing pneumonitis in a man with primary biliary cirrhosis, CREST syndrome and chronic pancreatitius. Thorax 1983, 38: 316-317. 